\title{
CONTROL EN LA FRONTERA LIBRE DE UNA VIGA VISCOELÁSTICA CON MEMORIA
}

\author{
Yolanda Silvia Santiago Ayala ${ }^{1}$ \& Santiago César Rojas Romero ${ }^{2}$
}

Resumen: Usando la Teoría de Semigrupo probamos la existencia de solución global de la ecuación de la viga con memoria, esta viga esta fija en uno de los extremos y en el otro dotado de un control $f$. También, usando técnicas multiplicativas, funcional de Lyapunov, sistemas dinámicos y algunos resultados de Datko, Buck, mostramos que la energía asociada al sistema decae a cero cuando $t \rightarrow+\infty$.

Palabras clave: Viga viscoelástica, Teoría de Control, Estabilización de la viga.

\section{CONTROL ON THE FREE BOUNDARY OF A VISCOELASTIC BEAN WITH MEMORY}

\begin{abstract}
Using Semigroups Theory, we prove the existence of global solution to the viscoelastic bean equation with memory, this bean is having one side fixed and the other one is provided of a control $f$. Also, using multiplication techniques, Lyapunov functional, dynamic systems and some results of Datko, Buck, we show that the energy associated to the system decays to zero when $t \rightarrow+\infty$, that means we get the stability of the bean.
\end{abstract}

Key words: Viscoelastic bean, Control Theory, Stability of the bean.

\section{Introducción}

Consideremos la viga viscoelástica con memoria de longitud finita $L$, que se encuentra presa en $x=0$ y libre en el otro extremo, en el cual suministraremos un control $f$. El material de la viga es isotrópico e incompresible satisfaciendo las ecuaciones constitutivas tipo Boltzmann. La ecuación del movimiento que gobierna el proceso es

$$
\begin{aligned}
& q u_{t t}-\left[r u_{x x}\right]_{x x}+a^{\prime} *\left[r u_{x x}\right]_{x x}=0,(x, t) \in(0, L) \times \mathbb{R}^{+} \\
& u(0, t)=u_{x}(0, t)=0, t \in \mathbb{R}^{+} \\
& {\left[r u_{x x}\right](L, t)+\left[a^{\prime} * r u_{x x}\right](L, t)=0, t \in \mathbb{R}^{+}} \\
& {\left[r u_{x x}\right]_{x}(L, t)+\left[a^{\prime} *\left(r u_{x x}\right)_{x}\right](L, t)=f(t), t \in \mathbb{R}^{+}} \\
& u(x, t)=w_{o}(x, t), \quad(x, t) \in[0, L] \times(-\infty, 0) \\
& u(x, 0)=u_{o}(x), u_{t}(x, 0)=u_{1}(x), x \in[0, L]
\end{aligned}
$$

donde, estamos adoptando la hipótesis de Bernoulli, i.e. eliminamos la inercia rotacional de las secciones transversales. $u(x, t)$ representan las desviaciones del eje neutral.

$q(x), r(x)$ y $a(t)$ son respectivamente, la densidad lineal, la rigidez flexural y el núcleo de relajación que representa la memoria del material. Consideraremos $r$ y $q$ medible y esencialmente positivo

\footnotetext{
${ }^{1}$ UNMSM, Facultad de Ciencias Matemáticas, e-mail: ysantiagoa@ummsm.edu.pe

${ }^{2}$ UNMSM, Facultad de Ciencias Matemáticas, e-mail: srojasr@unmsm.edu.pe
} 
en $[0, L], r \in L_{1}(0, L), \frac{1}{r} \in L_{\infty}$ y $\frac{1}{q} \in L_{1}(0, L)$.

El Operador * representa la convolución en el tiempo, esto es:

$$
a^{\prime} * b(L, t):=\int_{0}^{+\infty} a^{\prime}(s) b(L, t-s) d s
$$

Asumiremos a su vez que $a$ es un núcleo regular finito triplemente convexo, i.e.

(i) $a \in C^{2}[0,+\infty)$

(ii) $a(t) \geq 0, a^{\prime}(t) \leq 0, a^{\prime \prime}(t) \geq 0, \forall t \geq 0$.

(iii) $a(+\infty):=a_{\infty}>0, a(0)=1$

(iv) $a(t)-a_{\infty} \in L_{1}(0,+\infty)$

Estudiaremos la existencia de solución global y la estabilización de la viga del presente modelo. Para hacer el estudio de la existencia de solución usaremos la Teoría de Semigrupos (sección 3). Para la estabilización de la viga con control en la frontera, explícitamente nos interesa que la energía viscoelástica $E(t)$ tienda para cero cuando $t \rightarrow+\infty$, esto depende mucho también de $a$ la memoria del material, a través del cual se puede ver reflejada la tasa con que decae, lo hacemos en la sección 4, donde usamos en principio técnicas multiplicativas, funcional de Lyapunov, sistemas dinámicos y algunos resultados de Datko [4].

Uno de los trabajos pioneros en esta dirección son los de Dafermos [1], [2], [3] quien aborda en detalle la existencia, unicidad y estabilidad asintótica de la solución para ecuaciones mas generales de segundo orden. También debemos citar los trabajos [6], [9], [11], [14]. Problemas de incremento de disipación de la energía en función de una realimentación de control en la frontera, podemos encontrar en [7], [8], [10], donde son considerados otro tipo de núcleo, como por ejemplo. $[\exp (w t)](a(t)-d *)$ ó $[\exp (w t)] a^{\prime}(t)$, en L1, con $w>0$. Por otro lado, Desch and Miller [8] prueban que la desviación $u(t)$ y la velocidad $u^{\prime}(t)$ tienden a cero uniformemente con tasa exponencial siempre que $a(t)$ tienda a cero exponencialmente. De acuerdo al trabájo de MacCamy and Wong [12] podríamos afirmar que si $a(t)-d *$ no converge a cero exponencialmente, nuestro problema (1)-(3) no podría estabilizarse uniformemente y exponencialmente. Podemos citar, también, los trabajos de J. Rivera and M. Naso [13], Y. Santiago and J. Rivera [16] que ilustran como abordar el comportamiento asintótico de sistemas con memória. Oras técnicas para el comportamiento asintótico pueden ser vistas en [15], [17]. Tópicos interesantes de Análisis Funcional ligadas con las ecuaciones y conectadas a este estudio pueden ser vistos, por ejemplo, en [18].

\section{Resultado Principal}

Teorema 2.1. Sea $m$ una función suave en $(0, L)$ tal que $m(0)=0, m^{\prime \prime} \geq 0$, y $r$, $q$ dados al inicio, $m r, m^{\prime} r, m^{\prime \prime} r, m q$ son absolutamente continuas y esencialmente acotadas, $y$

(i) $p:=2 m^{\prime} r-\frac{1}{2}(m r)^{\prime}-\frac{1}{2} L \int_{0}^{L}\left(m^{\prime \prime} r\right) d x \frac{1}{r}>1$ casi siempre en $[0, L]$,

(ii) $\frac{1}{2}(m q)^{\prime}+\left(1-a_{\infty}\right) q>\xi$ casi siempre en $[0, L]$ para algún $\xi>0$.

Supongamos que el dato inicial $w_{0}(s)=u(-s)$ satisface

$$
w_{0}(\cdot) \in L_{2}\left(\mathbb{R}^{+}, a^{\prime}, V\right) \cap L_{1}\left(\mathbb{R}^{+}, a(\cdot)-a_{\infty}, V\right)
$$


$y\left(u_{0}, v_{0}\right) \in V \times H$. Además de las condiciones (i)-(iv) para a $(\cdot)$ dadas al inicio de este trabajo, asumiremos que

$$
\int_{0}^{\infty}\left(a(t)-a_{\infty}\right) t^{j} d t<\infty
$$

Entonces la energía viscoelástica $E(t)$, satisface

(a) $\int_{0}^{\infty} t^{j+1} E(t) d t<\infty$.

Si $e^{w t}\left(a(t)-a_{\infty}\right) \in L_{1}\left(\mathbb{R}^{+}\right)$o si $a(\cdot)-a_{\infty}=0$, entonces existen constantes $G>1, \alpha>0$ tal que (b) $E(t) \leq G e^{-\alpha t} E(0)$.

\section{Existencia y unicidad de solución}

Definimos:

$$
H:=L_{2}(0, L, q)
$$

que viene a ser el espacio- $L_{2}$ con peso (Energía Cinética ).

También definamos

$$
V:=\left\{u \in H, u(0)=u^{\prime}(0)=0, r u^{\prime \prime} \in \dot{H}\right\}
$$

que representa el espacio de la Energía Potencial, y

$$
W:=L_{2}\left(\mathbb{R}^{+}, a^{\prime}, V\right)
$$

es el espacio de las Distribuciones $V$-valuadas: $w($.$) de modo que \|w(.)\|_{V} \in L_{2}\left(\mathbb{R}^{+}, a^{\prime}\right)$.

Defina el Operador $L$ en $H$ como

$$
\begin{aligned}
L: D(L) & \rightarrow H \\
u & \rightarrow L u=\frac{1}{q}\left(r u^{\prime \prime}\right)^{\prime \prime}
\end{aligned}
$$

con dominio

$$
D(L)=\left\{u \in H, u, u^{\prime}, r u^{\prime \prime},\left(r u^{\prime \prime}\right)^{\prime} \text { son absolutamente continuas tal que } \frac{1}{q}\left(r u^{\prime \prime}\right)^{\prime \prime} \in H\right\} .
$$

Así, $L$ es maximal.

Si $a=0$ y $f=0$ caemos en el caso puramente elástico $a^{\prime}=0$ en $\mathbb{R}^{+}$. Esto es

$$
\begin{aligned}
& q u_{t t}-\left[r u_{x x}\right]_{x x}=0, \quad(x, t) \in(0, L) \times \mathbb{R}^{+} \\
& u(0, t)=u_{x}(0, t)=0, t \in \mathbb{R}^{+} \\
& {\left[r u_{x x}\right](L, t)=0, t \in \mathbb{R}^{+}} \\
& {\left[r u_{x x}\right]_{x}(L, t)=0, t \in \mathbb{R}^{+}} \\
& u(x, t)=w_{o}(x, t), \quad(x, t) \in[0, L] \times(-\infty, 0) \\
& u(x, 0)=u_{o}(x), u_{t}(x, 0)=u_{1}(x), x \in[0, L]
\end{aligned}
$$

De (3.1) obtenemos que $-u_{t t}=\left[r u_{x x}\right]_{x x} / q$.

La conservación de la energía elástica del sistema (3.1)-(3.4) conduce a definir el Operador $A_{H}$ como $A_{H}=L_{\left.\right|_{D\left(A_{H}\right)}}$, i.e. $A_{H} u:=L u, \forall u \in D\left(A_{H}\right)$, donde $D\left(A_{H}\right)=\{u \in D(L)$ tal que $u(0)=$ $\left.u^{\prime}(0)=\left(r u^{\prime \prime}\right)(L)=\left(r u^{\prime \prime}\right)^{\prime}(L)=0\right\}$ y así $A_{H}$ es Autoadjunta y positiva.

Obsérvese que $A_{H} u=-u_{t t}$ y que (3.2)-(3.4) están absorbidas en $D\left(A_{H}\right)$.

En efecto $A_{H}$ es Autoadjunta y Positiva, 
1. $<A_{H} u, u>_{H} \geq 0, \forall u \in D\left(A_{H}\right)$.

$$
\begin{aligned}
<A_{H} u, u>_{H} & =<\frac{1}{q}\left(r u^{\prime \prime}\right)^{\prime \prime}, u>_{H}=\int_{0}^{L} \frac{1}{q}\left(r u^{\prime \prime}\right)^{\prime \prime} u q d x \\
& =\int_{0}^{L}\left(r u^{\prime \prime}\right)^{\prime \prime} u d x=\left.u\left(r u^{\prime \prime}\right)^{\prime}\right|_{0} ^{L}-\int_{0}^{L}\left(r u^{\prime \prime}\right)^{\prime} u^{\prime} d x \\
& =u(L)\left(r u^{\prime \prime}\right)^{\prime}(L)-u(0)\left(r u^{\prime \prime}\right)^{\prime}(0)-\int_{0}^{L}\left(r u^{\prime \prime}\right)^{\prime} u^{\prime} d x \\
& =-\left.\left(r u^{\prime \prime}\right) u^{\prime}\right|_{0} ^{L}+\int_{0}^{L}\left(r u^{\prime \prime}\right) u^{\prime \prime} d x \\
& =-\left(r u^{\prime \prime}\right)(L) u^{\prime}(L)+\left(r u^{\prime \prime}\right)(0) u^{\prime}(0)+\int_{0}^{L}\left(r u^{\prime \prime}\right) u^{\prime \prime} d x \\
& =\int_{0}^{L} r\left(u^{\prime \prime}\right)^{2} d x \geq 0
\end{aligned}
$$

2. $<A_{H} u, v>_{H}=<u, A_{H} v>_{H}, \forall u, v \in D\left(A_{H}\right)$.

$$
\begin{aligned}
<A_{H} u, v>_{H} & =<\frac{1}{q}\left(r u^{\prime \prime}\right)^{\prime \prime}, v>_{H}=\int_{0}^{L} \frac{1}{q}\left(r u^{\prime \prime}\right)^{\prime \prime} v q d x \\
& =\int_{0}^{L}\left(r u^{\prime \prime}\right)^{\prime \prime} v d x=\left.\left(r u^{\prime \prime}\right) v\right|_{0} ^{L}-\int_{0}^{L}\left(r u^{\prime \prime}\right)^{\prime} v^{\prime} d x \\
& =\left(r u^{\prime \prime}\right)(L) v(L)-\left(r u^{\prime \prime}\right)^{\prime}(0) v(0)-\int_{0}^{L}\left(r u^{\prime \prime}\right)^{\prime} v^{\prime} d x \\
& =-\left.r u^{\prime \prime} v^{\prime}\right|_{0} ^{L}+\int_{0}^{L}\left(r u^{\prime \prime}\right) v^{\prime \prime} d x \\
& =-\left(r u^{\prime \prime}\right)(L) v^{\prime}(L)-\left(r u^{\prime \prime}\right)(0) v^{\prime}(0)+\int_{0}^{L} r u^{\prime \prime} v^{\prime \prime} d x \\
& =\int_{0}^{L} r u^{\prime \prime} v^{\prime \prime} d x
\end{aligned}
$$

Análogamente, intercambiando $u$ por $v$ tenemos:

$$
<A_{H} v, u>_{H}=\int_{0}^{L} r v^{\prime \prime} u^{\prime \prime} d x
$$

luego $<A_{H} u, v>_{H}=<A_{H} v, u>_{H}=<u, A_{H} v>_{H}$.

3. $D\left(A_{H}\right)=D\left(A_{H}^{*}\right)$

Ahora, usaremos el Operador $A_{H}$ para interpretar en sentido variacional las ecuaciones (1.1)-(1.6), y luego lo expresaremos como un Problema Abstracto en $V^{*}$.

Problema variacional:

$$
u_{t t}+A u+a^{\prime} * A u(t)+f \delta_{L}=0
$$

donde $A$ es la extención de $A_{H}, \delta_{L}$ es el $\delta$ de Dirac concentrada en $x=L$.

Por otro lado definimos el siguiente Operador $C$

$$
\begin{aligned}
C: V & \longrightarrow \mathbb{R} \\
\varphi & \longrightarrow \varphi(L)
\end{aligned}
$$


entonces $C$ es lineal y acotada.

Por lo tanto $C$ admite una aplicación adjunta acotada: $C^{*}$

$$
\begin{aligned}
C^{*}: \mathbb{R} & \longrightarrow V^{*} \\
r & \longrightarrow C^{*} r=r \delta_{L}
\end{aligned}
$$

En efecto, $C^{*} r(\varphi)=r(c \varphi)=r \varphi(L)=r \delta_{L}(\varphi)$.

Suponiendo que se conoce la velocidad en $x=L: u_{t}(L)$, podemos considerar el control:

$$
f(t):=k u_{t}(L)
$$

con $k>0$.

Luego, $f(t)=k C u_{t}$ y $f(t) \delta_{L}=\left(k C u_{t}\right) \delta_{L}=k\left(C u_{t} \delta_{L}\right)=k C^{*} C u_{t}$.

Por lo tanto la ecuación (3.7) puede ser expresada como:

$$
u_{t t}+A u+\int_{0}^{+\infty} a^{\prime}(s) A u(t-s) d s+k C^{*} C u_{t}=0, t>0
$$

Introducimos las siguientes variables:

$$
\begin{aligned}
w(x, t, s) & :=u(x, t-s), t>s \\
v(x, t) & :=u_{t}(x, t), t>0
\end{aligned}
$$

luego, el sistema (1.1)-(1.6) con el control feedback puede ser expresado del siguiente modo:

$$
\begin{gathered}
\frac{d}{d t}\left(\begin{array}{c}
u \\
v \\
w
\end{array}\right)=\left(\begin{array}{ccc}
0 & I & 0 \\
-A & -k C^{*} C & -\int_{0}^{+\infty} a^{\prime}(s) A(.) d s \\
0 & 0 & -D_{3}
\end{array}\right)\left(\begin{array}{c}
u \\
v \\
w
\end{array}\right)=\mathcal{A}\left(\begin{array}{c}
u \\
v \\
w
\end{array}\right) \\
\left(\begin{array}{c}
u \\
v \\
w
\end{array}\right)(0)=\left(\begin{array}{c}
u_{o} \\
v_{o} \\
w_{o}
\end{array}\right)
\end{gathered}
$$

Defina el espacio $E:=V \times H \times L_{2}\left(a^{\prime},[0,+\infty), V\right)=V \times H \times W$ con el producto interno

$$
\begin{gathered}
<(u, v, w),(\hat{u}, \hat{v}, \hat{w})>_{E}:=a_{\infty}<u, \hat{u}>_{V}+<v, \hat{v}>_{H}+ \\
\int_{0}^{+\infty}\left|a^{\prime}(s)\right|<u-w, \hat{u}-\hat{w}>_{V} d s \\
D(\mathcal{A})=\left\{(u, v, w) \in E, w(0)=u, w \in W_{2,1}\left(a^{\prime},(0, \infty), V\right)\right. \\
\left.A u+k C^{*} C u_{t}+\int_{0}^{\infty} a^{\prime}(s) A w d s \in H\right\}
\end{gathered}
$$

Ahora si, estamos listos para interpretar (3.12)--(3.13) como una ecuación de evolución en $E$ (Estamos siguiendo las ideas de Dafermos [3]). Para esto basta mostrar que el operador $\mathcal{A}$ con $D(\mathcal{A})$ sea disipativo maximal.

$$
\begin{aligned}
\frac{d}{d t} E(t) & =<\mathcal{A}(u, v, w),(u, v, w)>_{E} \\
& =-k v^{2}(L)-\frac{1}{2} \int_{0}^{\infty} a^{\prime \prime}(s) \underbrace{\|u-w(s)\|_{V}^{2}}_{\int_{0}^{x} r(u-w(s))_{x . x}^{2} d x} d s<0
\end{aligned}
$$




$$
\|\left(u(t), v(t), w(t)\left\|_{E}:=\right\| T(t)\left(u_{o}, v_{o}, w_{o}\right) \|_{E} \longrightarrow_{t \rightarrow+\infty} 0, \quad \forall\left(u_{o}, v_{o}, w_{o}\right) \in E\right.
$$

donde

$$
E(t)=\frac{1}{2}\|(u, v, w)\|_{E}^{2}
$$

Se satisface

$$
\begin{gathered}
D(\mathcal{A}) \supset \mathcal{D}:=\left\{(u, v, w) \in E \text { tal que } u \in D(L) \cap V, v \in V, w_{s} \in W, w(., 0)=u\right. \\
\left(r u_{x x}\right)(L)+\int_{0}^{+\infty} a^{\prime}(s)\left(r u_{x x}\right)(L, t-s) d s=0 \\
\left.\left(r u_{x x}\right)_{x}(L)+\int_{0}^{+\infty} a^{\prime}(s)\left(r u_{x x}\right)_{x}(L, t-s) d s=k v(L)\right\}
\end{gathered}
$$

donde la inclusión es densa, i.e. $\overline{\mathcal{D}}=D(\mathcal{A})$.

\section{Decaimiento de la Energía}

Sea $(u, v, w) \in D$, defina

$$
\begin{gathered}
W(t):=\int_{0}^{+\infty}\left|a^{\prime}(s)\right|(u(t)-u(t-s)) d s \\
G(t):=\left(m q\left(a_{\infty} u+W\right)_{x}-q W, u_{t}\right)
\end{gathered}
$$

donde $m$ es una función regular suave en $(0, L)$ con $m(0)=0$ de modo que $m r, m^{\prime} r, m^{\prime \prime} r, m q$ son absolutamente continuas y esencialmente acotadas.

Sabemos que

$$
\frac{d G}{d t}(t)=\underbrace{\left(m q\left(a_{\infty} u+W\right)_{x t}-q W_{t}, u_{t}\right)}_{F_{1}(t):=}+\underbrace{\left(m q\left(a_{\infty} u+W\right)_{x}-q W, u_{t t}\right)}_{F_{2}(t):=}
$$

donde

$$
\begin{aligned}
F_{1}(t)= & \int_{0}^{L}\left\{m q\left(a_{\infty} u_{x t}+W_{x t}\right)-q W_{t}\right\} u_{t} d x \\
= & \int_{0}^{L} m q a_{\infty} u_{x t} u_{t} d x+\int_{0}^{L} m q \int_{0}^{+\infty}\left|a^{\prime}(s)\right|\left(u_{x t}(t)-u_{x t}(t-s)\right) u_{t} d s d x \\
& -\int_{0}^{L} q W_{t} u_{t} d x \\
= & \underbrace{\int_{0}^{L} m q a_{\infty} u_{x t} u_{t} d x}_{I_{1}:=}+\underbrace{\int_{0}^{L} m q \int_{0}^{+\infty}\left|a^{\prime}(s)\right| u_{x t}(t) d s u_{t} d x}_{I_{2}:=} \\
& -\underbrace{-\int_{0}^{L} q W_{t} u_{t} d x}_{\left.\int_{0}^{L} m q \int_{0}^{+\infty}\left|a^{\prime}(s)\right| u_{x t}(t-s)\right) d s u_{t} d x}
\end{aligned}
$$




$$
\begin{aligned}
I_{1}+I_{2} & =\int_{0}^{L} m q a_{\infty} u_{x t} u_{t} d x-\int_{0}^{L} m q \int_{0}^{+\infty} a^{\prime}(s) d s u_{x t}(t) u_{t} d x \\
& =\int_{0}^{L} m q a_{\infty} u_{x t} u_{t} d x-\int_{0}^{L} m q\{\underbrace{a(+\infty)}_{a_{\infty}:=}-\underbrace{a(0)}_{1=}\} u_{x t}(t) u_{t} d x \\
& =\int_{0}^{L} m q u_{x t} u_{t} d x=\frac{1}{2} \int_{0}^{L} m q \frac{\partial}{\partial x}\left(u_{t}^{2}\right) d x \\
& =\frac{1}{2}\left\{\left(m q u_{t}^{2}\right)(L)-\left(m q u_{t}^{2}\right)(0)-\int_{0}^{L}(m q)^{\prime} u_{t}^{2} d x\right\} \\
& =\frac{1}{2}\left(m q u_{t}^{2}\right)(L)-\frac{1}{2} \int_{0}^{L}(m q)^{\prime} u_{t}^{2} d x
\end{aligned}
$$

$$
\begin{aligned}
I_{3}= & \int_{0}^{L} m q \int_{0}^{+\infty} a^{\prime}(s) u_{x t}(t-s) d s u_{t} d x \\
= & -\int_{0}^{L} m q \int_{0}^{+\infty} a^{\prime}(s) u_{x s}(t-s) d s u_{t} d x \\
= & -\int_{0}^{L} m q\left\{\left.a^{\prime}(s) u_{x}(t-s)\right|_{s=0} ^{+\infty}-\int_{0}^{+\infty} a^{\prime \prime}(s) u_{x}(t-s) d s\right\} u_{t} d x \\
= & -\int_{0}^{L} m q\left\{a^{\prime}(+\infty) u_{x}(t-+\infty)-a^{\prime}(0) u_{x}(t)\right\} u_{t} d x \\
& +\int_{0}^{L} m q \int_{0}^{+\infty} a^{\prime \prime}(s) u_{x}(t-s) d s u_{t} d x \\
= & a^{\prime}(0) \int_{0}^{L} m q u_{x}(t) u_{t} d x+\int_{0}^{L} m q \int_{0}^{+\infty} a^{\prime \prime}(s) u_{x}(t-s) d s u_{t} d x
\end{aligned}
$$




$$
\begin{aligned}
I_{4}= & -\int_{0}^{L} q W_{t} u_{t} d x \\
= & -\int_{0}^{L} q \int_{0}^{+\infty}\left|a^{\prime}(s)\right|(u(t)-u(t-s))_{t} d s u_{t} d x \\
= & \int_{0}^{L} q \int_{0}^{+\infty} a^{\prime}(s) u_{t}(t) d s u_{t} d x-\int_{0}^{L} q \int_{0}^{+\infty} a^{\prime}(s) u_{t}(t-s) d s u_{t} d x \\
= & \int_{0}^{L} q\left\{\int_{0}^{+\infty} a^{\prime}(s) d s\right\} u_{t}(t) u_{t} d x-\int_{0}^{L} q \int_{0}^{+\infty} a^{\prime}(s) u_{t}(t-s) d s u_{t} d x \\
= & -\left(1-a_{\infty}\right) \int_{0}^{L} q u_{t}^{2} d x-\int_{0}^{L} q \int_{0}^{+\infty} a^{\prime}(s) u_{t}(t-s) d s u_{t} d x \\
= & -\left(1-a_{\infty}\right) \int_{0}^{L} q u_{t}^{2} d x+\int_{0}^{L} q \int_{0}^{+\infty} a^{\prime}(s) u_{s}(t-s) d s u_{t} d x \\
= & -\left(1-a_{\infty}\right) \int_{0}^{L} q u_{t}^{2} d x+\int_{0}^{L} q\left\{\left.a^{\prime}(s) u(t-s)\right|_{s=0} ^{+\infty}-\int_{0}^{+\infty} a^{\prime \prime}(s) u(t-s) d s\right\} u_{t} d x \\
= & -\left(1-a_{\infty}\right) \int_{0}^{L} q u_{t}^{2} d x \\
& +\int_{0}^{L} q\{\underbrace{a^{\prime}(+\infty)}_{0=} u(t-+\infty)-a^{\prime}(0) u(t)-\int_{0}^{+\infty} a^{\prime \prime}(s) u(t-s) d s\} u_{t} d x \\
= & -\left(1-a_{\infty}\right) \int_{0}^{L} q u_{t}^{2} d x+\int_{0}^{L} q\left\{\int_{0}^{\infty} a^{\prime \prime}(s) d s u(t)-\int_{0}^{+\infty} a^{\prime \prime}(s) u(t-s) d s\right\} u_{t} d x \\
= & -\left(1-a_{\infty}\right) \int_{0}^{L} q u_{t}^{2} d x+\int_{0}^{L} q \int_{0}^{+\infty} a^{\prime \prime}(s)\{u(t)-u(t-s)\} d s u_{t} d x \\
= & -(x) \\
= & -(t) \\
= &
\end{aligned}
$$

Acomodando los términos de $I_{3}$, asociando las integrales y luego factorando tenemos

$$
\begin{aligned}
I_{3}+I_{4}= & -\int_{0}^{L} m q \frac{\partial}{\partial x}\left(\int_{0}^{+\infty} a^{\prime \prime}(s) u(t) d s\right) u_{t} d x+ \\
& \int_{0}^{L} m q \frac{\partial}{\partial x}\left(\int_{0}^{+\infty} a^{\prime \prime}(s) u(t-s) d s\right) u_{t} d x \\
& -\left(1-a_{\infty}\right) \int_{0}^{L} q u_{t}^{2} d x+\int_{0}^{L} q \int_{0}^{+\infty} a^{\prime \prime}(s)\{u(t)-u(t-s)\} d s u_{t} d x \\
= & \int_{0}^{L} m q \frac{\partial}{\partial x}\left(\int_{0}^{+\infty} a^{\prime \prime}(s)\{u(t-s)-u(t)\} d s\right) u_{t} d x \\
& -\left(1-a_{\infty}\right) \int_{0}^{L} q u_{t}^{2} d x-\int_{0}^{L} q \int_{0}^{+\infty} a^{\prime \prime}(s)\{u(t-s)-u(t)\} d s u_{t} d x \\
= & \int_{0}^{L} q\left(1-m \frac{\partial}{\partial x}\right)\left[\int_{0}^{+\infty} a^{\prime \prime}(s)\{u(t-s)-u(t)\} d s\right] u_{t} d x \\
& -\left(1-a_{\infty}\right) \int_{0}^{L} q u_{t}^{2} d x
\end{aligned}
$$

Ahora desde que $u \in V$ tenemos $u(x=0)=u_{x}(x=0)=0$, entonces de

$$
u(x)-u(0)=\int_{0}^{x} u_{x}(\xi) d \xi
$$


tenemos

$$
\begin{aligned}
|u(x)| & =\left|\int_{0}^{x} u_{x}(\xi) d \xi\right| \leq \int_{0}^{x}\left|u_{x}(\xi)\right| d \xi \leq \int_{0}^{L}\left|u_{x}(\xi)\right| d \xi=\int_{0}^{L} 1 \cdot\left|u_{x}(\xi)\right| d \xi \\
& \leq\left(\int_{0}^{L} 1 d \xi\right)^{\frac{1}{2}}\left(\int_{0}^{L}\left|u_{x}(\xi)\right|^{2} d \xi\right)^{\frac{1}{2}}=L^{\frac{1}{2}} \cdot\left(\int_{0}^{L}\left|u_{x}(\xi)\right|^{2} d \xi\right)^{\frac{1}{2}}
\end{aligned}
$$

Por lo tanto

$$
|u(x)|^{2} \leq L\left(\int_{0}^{L}\left|u_{x}(\xi)\right|^{2} d \xi\right)
$$

Análogamente

$$
\begin{gathered}
u_{x}(x)-u_{x}(0)=\int_{0}^{x} u_{x x}(\xi) d \xi \\
\left|u_{x}(x)\right|=\left|\int_{0}^{x} u_{x x}(\xi) d \xi\right| \leq \int_{0}^{x}\left|u_{x x}(\xi)\right| d \xi \leq \int_{0}^{L}\left|u_{x x}(\xi)\right| d \xi=\int_{0}^{L} 1 \cdot\left|u_{x x}(\xi)\right| d \xi \\
\leq\left(\int_{0}^{L} 1 d \xi\right)^{\frac{1}{2}}\left(\int_{0}^{L}\left|u_{x x}(\xi)\right|^{2} d \xi\right)^{\frac{1}{2}}=L^{\frac{1}{2}} \cdot\left(\int_{0}^{L}\left|u_{x x}(\xi)\right|^{2} d \xi\right)^{\frac{1}{2}}
\end{gathered}
$$

Por lo tanto

$$
\left|u_{x}(x)\right|^{2} \leq L\left(\int_{0}^{L}\left|u_{x x}(\xi)\right|^{2} d \xi\right)
$$

lo cual implica

$$
\begin{aligned}
\left|\left(1-m \frac{\partial}{\partial x}\right) u\right| & =\left|u-m u_{x}\right|=\left|\int_{0}^{x} u_{x}-m^{\prime} u_{x}-m u_{x x} d \xi\right| \\
& \leq \int_{0}^{x}\left|u_{x}-m^{\prime} u_{x}-m u_{x x}\right| d \xi \leq \int_{0}^{L}\left|u_{x}-m^{\prime} u_{x}-m u_{x x}\right| \cdot d \xi \\
& \leq \int_{0}^{L}\left|\left(1-m^{\prime}\right) u_{x}\right| d \xi+\int_{0}^{L}\left|m u_{x x}\right| d \xi \\
& \leq L^{\frac{1}{2}} \cdot\left(\int_{0}^{L}\left|\left(1-m^{\prime}\right) u_{x}\right|^{2} d \xi\right)^{\frac{1}{2}}+L^{\frac{1}{2}} \cdot\left(\int_{0}^{L}\left|m u_{x x}\right|^{2} d \xi\right)^{\frac{1}{2}} \\
& \leq L^{\frac{1}{2}} \cdot\left\{\left(\int_{0}^{L}\left|\left(1-m^{\prime}\right) u_{x}\right|^{2} d \xi\right)^{\frac{1}{2}}+\left(\int_{0}^{L}\left|m u_{x x}\right|^{2} d \xi\right)^{\frac{1}{2}}\right\}
\end{aligned}
$$

Por lo tanto

$$
\begin{aligned}
&\left|\left(1-m \frac{\partial}{\partial x}\right) u\right|^{2} \leq L \cdot\left\{\left(\int_{0}^{L}\left|\left(1-m^{\prime}\right) u_{x}\right|^{2} d x\right)^{\frac{1}{2}}+\left(\int_{0}^{L}\left|m u_{x x}\right|^{2} d x\right)^{\frac{1}{2}}\right\}^{2} \\
& \leq 2 L\{\underbrace{\int_{0}^{L}\left|\left(1-m^{\prime}\right) u_{x}\right|^{2} d x}_{*_{1}=}+\underbrace{\int_{0}^{L}\left|m u_{x x}\right|^{2} d x}_{*_{2}=}\} \\
& *_{2}=\int_{0}^{L}|m|^{2}\left|u_{x x}\right|^{2} d x \leq \int_{0}^{L} \sup m^{2}\left|u_{x x}\right|^{2} d x \leq \sup m^{2} \int_{0}^{L}\left|u_{x x}\right|^{2} d x
\end{aligned}
$$




$$
*_{1}=\int_{0}^{L}\left|\left(1-m^{\prime}\right) u_{x}\right|^{2} d x=\int_{0}^{L}\left|\left(1-m^{\prime}\right)\right|^{2}\left|u_{x}\right|^{2} d x \leq \sup \left(1-m^{\prime}\right)^{2} \int_{0}^{L}\left|u_{x}\right|^{2} d x
$$

pero

$$
\left|u_{x}(\xi)\right| \leq \int_{0}^{L}\left|u_{x x}(\xi)\right| d \xi \leq L^{\frac{1}{2}}\left(\int_{0}^{L}\left|u_{x x}(\xi)\right|^{2} d \xi\right)^{\frac{1}{2}}
$$

luego

$$
\left|u_{x}(\xi)\right|^{2} \leq L \int_{0}^{L}\left|u_{x x}(\xi)\right|^{2} d \xi
$$

integrando

$$
\begin{gathered}
\int_{0}^{L}\left|u_{x}(\xi)\right|^{2} d \xi \leq L^{2} \int_{0}^{L}\left|u_{x x}(\xi)\right|^{2} d \xi \\
*_{1} \leq L^{2} \sup \left(1-m^{\prime}\right)^{2} \int_{0}^{L}\left|u_{x x}(\xi)\right|^{2} d \xi
\end{gathered}
$$

Finalmente tenemos

$$
\left|\left(1-m \frac{\partial}{\partial x}\right) u\right|^{2} \leq \underbrace{2 L\left\{L^{2} \sup \left(1-m^{\prime}\right)^{2}+\sup m^{2}\right\}}_{\gamma_{o}=} \int_{0}^{L}\left|u_{x x}(\xi)\right|^{2} d \xi .
$$

Regresando al primer sumando de $I_{3}+I_{4}$ y usando que $a b \leq \frac{1}{2}\left(a^{2}+b^{2}\right)$ tenemos

$$
\begin{aligned}
& \left|\int_{0}^{L} \int_{0}^{+\infty} a^{\prime \prime}(s) q\left(1-m \frac{\partial}{\partial x}\right)\{u(t-s)-u(t)\} d s u_{t} d x\right| \\
& \leq \frac{1}{\sqrt{\nu}}\left\{\int_{0}^{L}\left(\int_{0}^{+\infty} a^{\prime \prime}(s)\left(1-m \frac{\partial}{\partial x}\right)\{u(t-s)-u(t)\} d s\right)^{2} d x\right\}^{\frac{1}{2}} \\
& \cdot \sqrt{\nu}\left\{\int_{0}^{L} q^{2} u_{t}^{2} d x\right\}^{\frac{1}{2}} \\
& \leq \frac{1}{2 \nu} \int_{0}^{L} \underbrace{\left(\int_{0}^{+\infty} a^{\prime \prime}(s)\left(1-m \frac{\partial}{\partial x}\right)\{u(t-s)-u(t)\} d s\right)^{2}}_{J_{1}=} d x \\
& +\frac{\nu}{2} \int_{0}^{L} q^{2} u_{t}^{2} d x \\
& J_{1} \leq\left[\int_{0}^{+\infty} a^{\prime \prime}(s) \cdot \gamma_{o}^{\frac{1}{2}}\left(\int_{0}^{L}\left|(u(t-s)-u)_{x x}\right|^{2} d x\right)^{\frac{1}{2}} d s\right]^{2} \\
& =\gamma_{o}\left[\int_{0}^{+\infty} a^{\prime \prime}(s)\left(\int_{0}^{L}\left|(u(t-s)-u)_{x x}\right|^{2} d x\right)^{\frac{1}{2}} d s\right]^{2} \\
& \leq \gamma_{o} \underbrace{\left(\int_{0}^{+\infty} a^{\prime \prime}(s) d s\right)}_{a^{\prime}(+\infty)-a^{\prime}(0)} \cdot \int_{0}^{+\infty} a^{\prime \prime}(s) \int_{0}^{L}\left|(u(t-s)-u)_{x x}\right|^{2} d x d s \\
& =\gamma_{o}\left[-a^{\prime}(0)\right] \int_{0}^{+\infty} a^{\prime \prime}(s) \int_{0}^{L}\left|(u(t-s)-u)_{x x}\right|^{2} d x d s \\
& =\gamma_{o}\left|a^{\prime}(0)\right| \int_{0}^{+\infty} a^{\prime \prime}(s) \int_{0}^{L}\left|(u(t-s)-u)_{x x}\right|^{2} d x d s
\end{aligned}
$$


Utilizando la mayoración de $J_{1}$ tenemos

$$
\begin{aligned}
& \left|\int_{0}^{L} \int_{0}^{+\infty} a^{\prime \prime}(s) q\left(1-m \frac{\partial}{\partial x}\right)\{u(t-s)-u(t)\} d s u_{t} d x\right| \\
\leq & \frac{\nu}{2} \int_{0}^{L} q^{2} u_{t}^{2} d x+\frac{1}{2 \nu} \int_{0}^{L} \underbrace{\gamma\left|a^{\prime}(0)\right| \int_{0}^{+\infty} a^{\prime \prime}(s) \int_{0}^{L}\left|(u(t-s)-u)_{x x}\right|^{2} d x d s}_{\text {no depende de } x} d x \\
= & \frac{\nu}{2} \int_{0}^{L} q^{2} u_{t}^{2} d x+\frac{L}{2 \nu} \gamma_{o}\left|a^{\prime}(0)\right| \int_{0}^{+\infty} a^{\prime \prime}(s)\left[\int_{0}^{L}\left|(u(t-s)-u)_{x x}\right|^{2} d x\right] d s
\end{aligned}
$$

donde $\nu \in(0,1)$.

Así mayoramos $F_{1}(t)$ :

$$
\begin{aligned}
F_{1}(t)= & \left(I_{1}+I_{2}\right)+\left(I_{3}+I_{4}\right) \\
\leq & \frac{1}{2}\left(m q u_{t}^{2}\right)(L)-\frac{1}{2} \int_{0}^{L}(m q)^{\prime} u_{t}^{2} d x-\left(1-a_{\infty}\right) \int_{0}^{L} q u_{t}^{2} d x \\
& +\frac{\nu}{2} \int_{0}^{L} q^{2} u_{t}^{2} d x+\frac{L}{2 \nu} \gamma_{o}\left|a^{\prime}(0)\right| \int_{0}^{+\infty} a^{\prime \prime}(s)\left[\int_{0}^{L}\left|(u(t-s)-u)_{x x}\right|^{2} d x\right] d s \\
= & \frac{1}{2}\left(m q u_{t}^{2}\right)(L)-\int_{0}^{L}\left\{\frac{1}{2}(m q)^{\prime}+\left(1-a_{\infty}\right) q-\frac{\nu}{2} q^{2}\right\} u_{t}^{2} d x \\
& +\frac{L}{2 \nu} \gamma_{o}\left|a^{\prime}(0)\right| \sup \frac{1}{r} \int_{0}^{+\infty} a^{\prime \prime}(s)\left[\int_{0}^{L} r(x)\left|(u(t-s)-u)_{x x}\right|^{2} d x\right] d s \\
= & \frac{1}{2}\left(m q u_{t}^{2}\right)(L)-\int_{0}^{L}\left\{\frac{1}{2}(m q)^{\prime}+\left(1-a_{\infty}\right) q-\frac{\nu}{2} q^{2}\right\} u_{t}^{2} d x \\
& +\frac{L}{2 \nu} \gamma_{o}\left|a^{\prime}(0)\right| \sup \frac{1}{r} \int_{0}^{+\infty} a^{\prime \prime}(s)\|(u(t-s)-u)\|_{V}^{2} d s
\end{aligned}
$$

Ahora procedemos a mayorar $F_{2}(t)$, usando la ecuación (1.1), integración por partes, introduciendo $\pm\left(r u_{x x}(t)\right)_{x} \int_{0}^{\infty} a^{\prime}(s) d s= \pm\left(r u_{x x}(t)\right)_{x}\{\underbrace{a(+\infty)}_{a=\infty}-\underbrace{a(0)}_{=1}\}$, ecuación $(1.4)$ con $f(t)=k u_{t}(L) W(0)=$ 
0 , pues $u(0)=0=m(0)$ y ecuación (1.3),(1.2) respectivamente tenemos:

$$
\begin{aligned}
& F_{2}(t):=\left(m q\left(a_{\infty} u+W\right)_{x}-q W, u_{t t}\right)=\left(\left\{m\left(a_{\infty} u+W\right)_{x}-W\right\} q, u_{t t}\right) \\
& =\int_{0}^{L}\left\{m\left(a_{\infty} u+W\right)_{x}-W\right\} q \cdot u_{t t} d x \\
& =-\int_{0}^{L}\left\{m\left(a_{\infty} u+W\right)_{x}-W\right\} \cdot\left\{\left(r u_{x x}\right)_{x x}+\int_{0}^{+\infty} a^{\prime}(s)\left(r u_{x x}(t-s)\right)_{x x} d s\right\} d x \\
& =-\left.\left\{m\left(a_{\infty} u+W\right)_{x}-W\right\} \cdot\left\{\left(r u_{x x}\right)_{x}+\int_{0}^{+\infty} a^{\prime}(s)\left(r u_{x x}(t-s)\right)_{x} d s\right\}\right|_{0} ^{L} \\
& +\int_{0}^{L}\left\{m\left(a_{\infty} u+W\right)_{x}-W\right\}_{x} \cdot\left\{\left(r u_{x x}\right)_{x}+\int_{0}^{+\infty} a^{\prime}(s)\left(r u_{x x}(t-s)\right)_{x} d s\right\} d x \\
& =-\left.\left\{m\left(a_{\infty} u+W\right)_{x}-W\right\} \cdot\left\{\left(r u_{x x}\right)_{x}+\int_{0}^{+\infty} a^{\prime}(s)\left(r u_{x x}(\dot{t}-s)\right)_{x} d s\right\}\right|_{0} ^{L} \\
& +\int_{0}^{L}\left\{m\left(a_{\infty} u+W\right)_{x}-W\right\}_{x} \cdot\left\{\int_{0}^{+\infty} a^{\prime}(s)\left(r(u(t-s)-u)_{x x}\right)_{x} d s\right\} d x \\
& +\int_{0}^{L}\left\{m\left(a_{\infty} u+W\right)_{x}-W\right\}_{x} \cdot\left\{\left(r u_{x x}\right)_{x} \int_{0}^{+\infty} a^{\prime}(s) d s+\left(r u_{x x}\right)_{x}\right\} d x \\
& =-\left.\left\{m\left(a_{\infty} u+W\right)_{x}-W\right\} \cdot\left\{\left(r u_{x x}\right)_{x}+\int_{0}^{+\infty} a^{\prime}(s)\left(r u_{x x}(t-s)\right)_{x} d s\right\}\right|_{0} ^{L} \\
& +\int_{0}^{L}\left\{m\left(a_{\infty} u+W\right)_{x}-W\right\}_{x} \cdot\left\{a_{\infty}\left(r u_{x x}\right)_{x}+\int_{0}^{+\infty} a^{\prime}(s)\left(r(u(t-s)-u)_{\dot{x} x}\right)_{x} d s\right\} d x \\
& =-\left\{m(L)\left(a_{\infty} u+W\right)_{x}(L)-W(L)\right\} k u_{t}(L)+0 \\
& +\int_{0}^{L}\left\{m\left(a_{\infty} u+W\right)_{x}-W\right\}_{x} \cdot\left\{a_{\infty}\left(r u_{x x}\right)_{x}+\left(r W_{x x}\right)_{x}\right\} d x \\
& =-\left\{m(L)\left(a_{\infty} u+W\right)_{x}(L)-W(L)\right\} k u_{t}(L) \\
& +\left.\left\{m\left(a_{\infty} u+W\right)_{x}-W\right\}_{x} \cdot\left\{a_{\infty} r u_{x x}+r W_{x x}\right\}\right|_{0} ^{L} \\
& -\int_{0}^{L}\left\{m\left(a_{\infty} u+W\right)_{x}-W\right\}_{x x} \cdot\left\{a_{\infty} r u_{x x}+r W_{x x}\right\} d x \\
& =-\left\{m(L)\left(a_{\infty} u+W\right)_{x}(L)-W(L)\right\} k u_{t}(L) \\
& -\int_{0}^{L}\left\{m\left(a_{\infty} u+W\right)_{x}-W\right\}_{x x} \cdot\left\{r\left(a_{\infty} u+W\right)_{x x}\right\} d x \\
& =\underbrace{-\left\{m(L)\left(a_{\infty} u+W\right)_{x}(L)-W(L)\right\} k u_{t}(L)}_{R_{1}:=} \\
& \underbrace{-\int_{0}^{L}\left\{m\left(a_{\infty} u+W\right)_{x}\right\}_{x x} \cdot\left\{r\left(a_{\infty} u+W\right)_{x x}\right\} d x}_{I_{5}:=} \\
& +\underbrace{\int_{0}^{L} r W_{x x}\left(a_{\infty} u+W\right)_{x x} d x}_{I_{6}:=}
\end{aligned}
$$




$$
\begin{aligned}
& I_{5}=-\int_{0}^{L}\left\{m\left(a_{\infty} u+W\right)_{x}\right\}_{x x} \cdot\left\{r\left(a_{\infty} u+W\right)_{x x}\right\} d x \\
& =-\int_{0}^{L}\left[\left\{m\left(a_{\infty} u+W\right)_{x x}\right\}_{x}+\left\{m^{\prime}\left(a_{\infty} u+W\right)_{x}\right\}_{x}\right] \cdot\left\{r\left(a_{\infty} u+W\right)_{x x}\right\} d x \\
& =-\int_{0}^{L} m^{\prime} r\left\{\left(a_{\infty} u+W\right)_{x x}\right\}^{2} d x-\int_{0}^{L} r m\left(a_{\infty} u+W\right)_{x x x}\left(a_{\infty} u+W\right)_{x x} d x \\
& -\int_{0}^{L} m^{\prime} r\left\{\left(a_{\infty} u+W\right)_{x x}\right\}^{2} d x-\int_{0}^{L} r m^{\prime \prime}\left(a_{\infty} u+W\right)_{x}\left(a_{\infty} u+W\right)_{x x} d x \\
& =-2 \int_{0}^{L} m^{\prime} r\left\{\left(a_{\infty} u+W\right)_{x x}\right\}^{2} d x-\frac{1}{2} \int_{0}^{L} m r \frac{\partial}{\partial x}\left\{\left(a_{\infty} u+W\right)_{x x}\right\}^{2} d x \\
& -\frac{1}{2} \int_{0}^{L} m^{\prime \prime} r \frac{\partial}{\partial x}\left\{\left(a_{\infty} u+W\right)_{x}\right\}^{2} d x \\
& =-2 \int_{0}^{L} m^{\prime} r\left\{\left(a_{\infty} u+W\right)_{x x}\right\}^{2} d x-\frac{1}{2}\left\{\left.r m\left\{\left(a_{\infty} u+W\right)_{x x}\right\}^{2}\right|_{0} ^{L}\right. \\
& \left.-\int_{0}^{L}(m r)^{\prime}\left\{\left(a_{\infty} u+W\right)_{x x}\right\}^{2} d x\right\} \\
& -\frac{1}{2}\left\{\left.m^{\prime \prime} r\left\{\left(a_{\infty} u+W\right)_{x}\right\}^{2}\right|_{0} ^{L}-\int_{0}^{L}\left(m^{\prime \prime} r\right)^{\prime}\left\{\left(a_{\infty} u+W\right)_{x}\right\}^{2} d x\right\} \\
& =-\int_{0}^{L}\left(2 m^{\prime} r-\frac{1}{2}(r m)^{\prime}\right)\left\{\left(a_{\infty} u+W\right)_{x x}\right\}^{2} d x \\
& -\frac{1}{2} r(L) m(L)\left\{\left(a_{\infty} u+W\right)_{x x}\right\}^{2}(L)-\frac{1}{2} r(L) m^{\prime \prime}(L)\left\{\left(a_{\infty} u+W\right)_{x}\right\}^{2}(L) \\
& +\frac{1}{2} \int_{0}^{L}\left(m^{\prime \prime} r\right)^{\prime}\left\{\left(a_{\infty} u+W\right)_{x}\right\}^{2} d x \\
& =-\int_{0}^{L}\left(2 m^{\prime} r-\frac{1}{2}(r m)^{\prime}\right)\left\{\left(a_{\infty} u+W\right)_{x x}\right\}^{2} d x \\
& -\frac{1}{2} r(L) m^{\prime \prime}(L)\left\{\left(a_{\infty} u+W\right)_{x}\right\}^{2}(L)+\frac{1}{2} \int_{0}^{L}\left(m^{\prime \prime} r\right)^{\prime}\left\{\left(a_{\infty} u+W\right)_{x}\right\}^{2} d x \\
& \leq-\int_{0}^{L}\left(2 m^{\prime} r-\frac{1}{2}(r m)^{\prime}\right)\left\{\left(a_{\infty} u+W\right)_{x x}\right\}^{2} d x \\
& +\frac{1}{2} \int_{0}^{L}\left(m^{\prime \prime} r\right)^{\prime}\left\{\left(a_{\infty} u+W\right)_{x}\right\}^{2} d x \\
& \leq-\int_{0}^{L}\left(2 m^{\prime} r-\frac{1}{2}(r m)^{\prime}-\frac{1}{2} L \int_{0}^{L}\left(m^{\prime \prime} r\right)^{\prime} d x\right)\left\{\left(a_{\infty} u+W\right)_{x x}\right\}^{2} d x
\end{aligned}
$$

considerando

$$
p:=\frac{2 m^{\prime} r-\frac{1}{2}(r m)^{\prime}-\frac{1}{2} L \int_{0}^{L}\left(m^{\prime \prime} r\right)^{\prime} d x}{r}
$$

entonces

$$
I_{5} \leq-\int_{0}^{L} \operatorname{pr}\left\{\left(a_{\infty} u+W\right)_{x x}\right\}^{2} d x
$$

La energía potencial esta dada por

$$
E_{p o t}:=\frac{1}{2}\left\{a_{\infty} \int_{0}^{L} r u_{x x}^{2} d x+\int_{0}^{+\infty}\left|a^{\prime}(s)\right| \int_{0}^{L} r\left[(u(t-s)-u)_{x x}\right]^{2} d x d s\right\}
$$


luego

$$
\begin{aligned}
& E_{\text {pot }}=\frac{1}{2}\left\{a_{\infty} \int_{0}^{L} r u_{x x}^{2} d x\right. \\
& \left.+\int_{0}^{+\infty}\left|a^{\prime}(s)\right| \int_{0}^{L} r\left[\left(u_{x x}(t-s)\right)^{2}+\left(u_{x x}\right)^{2}-2 u_{x x}(t-s) u_{x x}\right] d x d s\right\} \\
& =\frac{a_{\infty}}{2} \int_{0}^{L} r u_{x x}^{2} d x-\int_{0}^{+\infty}\left|a^{\prime}(s)\right| \int_{0}^{L} r u_{x x}(t-s) u_{x x} d x d s \\
& +\frac{1}{2} \int_{0}^{+\infty}\left|a^{\prime}(s)\right| \int_{0}^{L} r\left(u_{x x}(t-s)\right)^{2} d x d s+\frac{1}{2} \underbrace{\int_{0}^{+\infty}\left|a^{\prime}(s)\right| d s}_{=1-a_{\infty}} \int_{0}^{L} r\left(u_{x x}\right)^{2} d x \\
& =\frac{1}{2} \int_{0}^{L} r u_{x x}^{2} d x-\int_{0}^{+\infty}\left|a^{\prime}(s)\right| \int_{0}^{L} r u_{x x}(t-s) u_{x x} d x d s \\
& +\frac{1}{2} \int_{0}^{+\infty}\left|a^{\prime}(s)\right| \int_{0}^{L} r\left(u_{x x}(t-s)\right)^{2} d x d s \\
& =\frac{1}{2} \int_{0}^{L} r u_{x x}^{2} d x+\int_{0}^{+\infty} a^{\prime}(s) \int_{0}^{L} r u_{x x}(t-s) u_{x x} d x d s . \\
& -\frac{1}{2} \int_{0}^{+\infty} a^{\prime}(s) \int_{0}^{L} r\left(u_{x x}(t-s)\right)^{2} d x d s \\
& =\frac{1}{2} \int_{0}^{L} r u_{x x}^{2} d x+\int_{0}^{+\infty} a^{\prime}(s) \int_{0}^{L} r u_{x x}(t-s) u_{x x} d x d s \\
& -\frac{1}{2} \int_{-\infty}^{t} a^{\prime}(t-\varsigma) \int_{0}^{L} r\left(u_{x x}(\varsigma)\right)^{2} d x d \varsigma
\end{aligned}
$$

Considerando el primer y último término de la precedente igualdad y el siguiente resultado basado en una identidad de Buck

$$
\begin{aligned}
& \frac{\partial}{\partial t}[\int_{-\infty}^{t}\left\{a(t-\varsigma)-a_{\infty}\right\} \underbrace{\left(\int_{0}^{L} r u_{x x}^{2}(\varsigma) d x\right)}_{\hat{H}(\varsigma):=} d \varsigma] \\
& =\left\{a(t-t)-a_{\infty}\right\} \hat{H}(t)+\int_{-\infty}^{t} \frac{\partial}{\partial t}\left\{a(t-\varsigma)-a_{\infty}\right\} \hat{H}(\varsigma) d \varsigma \\
& =\left\{a(0)-a_{\infty}\right\} \hat{H}(t)+\int_{-\infty}^{t} a^{\prime}(t-\varsigma) \hat{H}(\varsigma) d \varsigma \\
& =\left\{1-a_{\infty}\right\} \int_{0}^{L} r u_{x x}^{2}(t) d x+\int_{-\infty}^{t} a^{\prime}(t-\varsigma) \int_{0}^{L} r u_{x x}^{2}(\varsigma) d x d \varsigma,
\end{aligned}
$$

tenemos que

$$
\begin{aligned}
E_{\text {pot }}= & \left\{1-\frac{a_{\infty}}{2}\right\} \int_{0}^{L} r u_{x x}^{2}(t) d x-\frac{1}{2} \frac{\partial}{\partial t}\left[\int_{-\infty}^{t}\left\{a(t-\varsigma)-a_{\infty}\right\}\left(\int_{0}^{L} r u_{x x}^{2}(\varsigma) d x\right) d \varsigma\right] \\
& +\int_{0}^{+\infty} a^{\prime}(s) \int_{0}^{L} r u_{x x}(t-s) u_{x x} d x d s .
\end{aligned}
$$


Por otro lado, usando la definición de $W$ y Fubini tenemos

$$
\begin{aligned}
I_{6}:= & \int_{0}^{L} r W_{x x}\left\{a_{\infty} u_{x x}+W_{x x}\right\} d x \\
= & a_{\infty} \int_{0}^{L} r W_{x x} u_{x x} d x+\int_{0}^{L} r\left(W_{x x}\right)^{2} d x \\
= & a_{\infty} \int_{0}^{L} r\left\{\int_{0}^{+\infty} a^{\prime}(s)(u(t-s)-u(t))_{x x} d s\right\} u_{x x} d x+\int_{0}^{L} r\left(W_{x x}\right)^{2} d x \\
= & a_{\infty} \int_{0}^{L} r \int_{0}^{+\infty} a^{\prime}(s) u_{x x}(t-s) d s u_{x x} d x-a_{\infty} \int_{0}^{L} r \underbrace{\left(\int_{0}^{+\infty} a^{\prime}(s) d s\right)}_{=a_{\infty}-1} u_{x x}^{2}(t) d x \\
& +\int_{0}^{L} r\left(W_{x x}\right)^{2} d x \\
= & a_{\infty} \int_{0}^{+\infty} a^{\prime}(s) \int_{0}^{L} r u_{x x}(t-s) u_{x x} d x d s+a_{\infty}\left(1-a_{\infty}\right) \int_{0}^{L} r u_{x x}^{2} d x \\
& +\int_{0}^{L} r\left(W_{x x}\right)^{2} d x
\end{aligned}
$$

Utilizando la definición de $W$ y Fubini en la desigualdad (4.2), tenemos

$$
\begin{aligned}
& I_{5} \leq-\int_{0}^{L} \operatorname{pr}\left\{a_{\infty} u_{x x}+W_{x x}\right\}^{2} d x \\
& =-\int_{0}^{L} \operatorname{pra}_{\infty}^{2}\left(u_{x x}\right)^{2} d x-2 \int_{0}^{L} \operatorname{pra}_{\infty} u_{x x} W_{x x} d x-\int_{0}^{L} \operatorname{pr}\left(W_{x x}\right)^{2} d x \\
& =-\int_{0}^{L} \operatorname{pra}_{\infty}^{2}\left(u_{x x}\right)^{2} d x-2 \int_{0}^{L} \operatorname{pra}_{\infty} u_{x x}\left\{\int_{0}^{+\infty} a^{\prime}(s)(u(t-s)-u(t))_{x x} d s\right\} d x \\
& -\int_{0}^{L} \operatorname{pr}\left(W_{x x}\right)^{2} d x \\
& =-\int_{0}^{L} \operatorname{pra}_{\infty}^{2}\left(u_{x x}\right)^{2} d x-2 \int_{0}^{L} \operatorname{pra}_{\infty} u_{x x}\left\{\int_{0}^{+\infty} a^{\prime}(s) u_{x x}(t-s) d s\right\} d x \\
& +2 a_{\infty}\left(a_{\infty}-1\right) \int_{0}^{L} \operatorname{pr} u_{x x}^{2} d x-\int_{0}^{L} \operatorname{pr}\left(W_{x x}\right)^{2} d x \\
& =\left\{-a_{\infty}^{2}+2 a_{\infty}\left(a_{\infty}-1\right)\right\} \int_{0}^{L} \operatorname{pr}\left(u_{x x}\right)^{2} d x-2 a_{\infty} \int_{0}^{+\infty} a^{\prime}(s) \int_{0}^{L} \operatorname{pru}_{x x}(t-s) u_{x x}(t) d x d s \\
& -\int_{0}^{L} \operatorname{pr}\left(W_{x x}\right)^{2} d x \\
& =-2 a_{\infty}\left(1-\frac{a_{\infty}}{2}\right) \int_{0}^{L} \operatorname{pr}\left(u_{x x}\right)^{2} d x-2 a_{\infty} \int_{0}^{+\infty} a^{\prime}(s) \int_{0}^{L} \operatorname{pr} u_{x x}(t-s) u_{x x}(t) d x d s \\
& -\int_{0}^{L} \operatorname{pr}\left(W_{x x}\right)^{2} d x
\end{aligned}
$$


Por lo tanto sumando las desigualdades y usando $-\inf (2 p-1) \geq-(2 p-1)$ tenemos

$$
\begin{aligned}
I_{6}+I_{5} \leq & -\int_{0}^{L}(p-1) r\left(W_{x x}\right)^{2} d x \\
& +\int_{0}^{+\infty}\left[a_{\infty}\left(1-a_{\infty}\right)-2 p a_{\infty}\left(1-\frac{1}{2} a_{\infty}\right)\right] r\left(u_{x x}\right)^{2} d x \\
& -a_{\infty} \int_{0}^{+\infty} a^{\prime}(s) \int_{0}^{L}(2 p-1) r u_{x x}(t-s) u_{x x} d x d s \\
= & -\int_{0}^{L}(p-1) r\left(W_{x x}\right)^{2} d x \\
& -\int_{0}^{+\infty}\left[a_{\infty}\left(1-\frac{1}{2} a_{\infty}\right)(2 p-1)+\frac{1}{2} a_{\infty}^{2}\right] r\left(u_{x x}\right)^{2} d x \\
& -a_{\infty} \int_{0}^{+\infty} a^{\prime}(s) \int_{0}^{L}(2 p-1) r u_{x x}(t-s) u_{x x} d x d s \\
\leq & -\int_{0}^{L}(p-1) r\left(W_{x x}\right)^{2} d x \\
& -\int_{0}^{+\infty}\left[a_{\infty}\left(1-\frac{1}{2} a_{\infty}\right)(2 p-1)+\frac{1}{2} a_{\infty}^{2}\right] r\left(u_{x x}\right)^{2} d x \\
& -a_{\infty} \operatorname{Inf}(2 p-1) \int_{0}^{+\infty} a^{\prime}(s) \int_{0}^{L} r u_{x x}(t-s) u_{x x} d x d s
\end{aligned}
$$

Por conveniencia, defina

$$
\begin{aligned}
\gamma_{1} & :=\inf (2 p-1) \\
p_{1} & :=a_{\infty}\left(1-\frac{1}{2} a_{\infty}\right)(2 p-1)+\frac{1}{2} a_{\infty}^{2}
\end{aligned}
$$

y usando la igualdad $E_{p o t}$, la desigualdad anterior queda expresado por:

$$
\begin{aligned}
I_{5}+I_{6} \leq & -\int_{0}^{L}(p-1) r\left(W_{x x}\right)^{2} d x-p_{1} \int_{0}^{+\infty} r\left(u_{x x}\right)^{2} d x \\
& -a_{\infty} \gamma_{1} \int_{0}^{+\infty} a^{\prime}(s) \int_{0}^{L} r u_{x x}(t-s) u_{x x} d x d s \\
= & -\int_{0}^{L}(p-1) r\left(W_{x x}\right)^{2} d x-p_{1} \int_{0}^{+\infty} r\left(u_{x x}\right)^{2} d x \\
& -a_{\infty} \gamma_{1}\left\{E_{p o t}-\left(1-\frac{a_{\infty}}{2}\right) \int_{0}^{L} r\left(u_{x x}\right)^{2} d x+\right. \\
& -\int_{0}^{L}(p-1) r\left(W_{x x}\right)^{2} d x-p_{1} \int_{0}^{+\infty} r\left(u_{x x}\right)^{2} d x \\
& -a_{\infty} \gamma_{1} E_{p o t}+a_{\infty} \gamma_{1}\left(1-\frac{a_{\infty}}{2}\right) \int_{0}^{L} r\left(u_{x x}\right)^{2} d x \\
& -\frac{a_{\infty} \gamma_{1}}{2} \frac{\partial}{\partial t} \int_{-\infty}^{t}\left(a(t-s)-a_{\infty}\right) \int_{0}^{L} r\left(u_{x x}(s)\right)^{2} d x d s
\end{aligned}
$$


Finalmente

$$
\begin{aligned}
R_{1} & =-\left\{m(L)\left(a_{\infty} u+W\right)_{x}(L)-W(L)\right\} k u_{t}(L) \\
& \leq \frac{\eta}{2} L m^{2}(L) k^{2} \int_{0}^{L}\left[a_{\infty}^{2} u_{x x}^{2}+(1+L) W_{x x}^{2}\right] d x+\frac{1}{2 \eta} u_{t}^{2}(L) \text { for } \eta \in(0, L)
\end{aligned}
$$

Así conseguimos estimar $F_{2}$ :

$$
\begin{aligned}
F_{2}(t) \leq & -\int_{0}^{L}\left[\left(p_{1}-a_{\infty} \gamma_{1}\left(1-\frac{a_{\infty}}{2}\right)\right)-\frac{\eta}{2} m^{2}(L) L k^{2} a_{\infty}\left(\frac{1}{r}\right)\right] r u_{x x}^{2} d x \\
& -a_{\infty} \gamma_{1} E_{p o t}-\int_{0}^{L}\left[(p-1) r-\frac{\eta}{2} m^{2}(L) k^{2} L(1+L)\right] W_{x x}^{2} d x \\
& -a_{\infty} \gamma_{1} \frac{1}{2} \frac{\partial}{\partial t} \int_{-\infty}^{t}\left(a(t-s)-a_{\infty}\right) \int_{0}^{L} r u_{x x}^{2}(s) d s d x+\frac{1}{2 \eta} u_{t}^{2}(L)
\end{aligned}
$$

\section{Asumiremos entonces:}

(i) $p-1>0$ casi siempre en $[0, L]$.

(ii) $\frac{1}{2}(m q)^{\prime}+\left(1-a_{\infty}\right) q>\xi$ casi siempre en $[0, L], \xi>0$.

Entonces, para $\nu, \eta$ suficientemente pequeño, tenemos que

$$
\begin{aligned}
\frac{d}{d t} G(t)= & F_{1}(t)+F_{2}(t) \leq\left(\frac{1}{2}(m q)(L)+\frac{1}{2 \eta}\right) u_{t}^{2}(L) \\
& +\gamma_{0} \sup \frac{1}{r} \frac{\left|a^{\prime}(0)\right|}{2 \nu} \int_{0}^{\infty} a^{\prime \prime}(s)\|u(t-s)-u\|_{V}^{2} d s-\min \left(\xi, a_{\infty} \gamma_{1}\right) E(t) \\
& -a_{\infty} \gamma_{1} \frac{1}{2} \frac{\partial}{\partial t} \int_{-\infty}^{t}\left(a(t-s)-a_{\infty}\right) \int_{0}^{L} r u_{x x}^{2}(s) d x d s
\end{aligned}
$$

Definimos

$$
F_{\epsilon}(t):=E(t)+\epsilon G(t)
$$

Luego

$$
\frac{d}{d t} F_{\epsilon}(t) \leq-\epsilon \min \left(\xi, a_{\infty} \gamma_{1}\right) E(t)-\epsilon a_{\infty} \gamma_{1} \frac{1}{2} \frac{\partial}{\partial t} \int_{-\infty}^{t}\left(a(t-s)-a_{\infty}\right) \int_{0}^{L} r u_{x x}^{2}(s) d x d s
$$

Por otro lado utilizando las mismas técnicas tenemos que existe $C>0$ tal que $G(t) \leq C E(t)$. Así, para $\epsilon$ suficientemente pequeño se tiene $(1-\epsilon C) E(t) \leq F_{\epsilon}(t) \leq(1+\epsilon C) E(t)$. 


\section{REFERENCIAS BIBLIOGRÁFICAS}

[1] C. M. Dafermos. As abstract Volterra equation with applications in linear viscoelasticity. J. Differential Equations 7 (1970) $554-569$.

[2] C. M. Dafermos. Asymptotic stability in viscoelasticity. Arch Rational Mech. Anal. 37 (1970) 297-308.

[3] C. M. Dafermos. Contraction semigroups and trend to equilibrium in continuum mechanics. In Applications of Methods of functional Analysis to Problems in Mechanics. Eds B. Nayroles and P. Germain, pp 295-306. Joint Symposium IUTAM/IMU (Berlin: Springer, 1976).

[4] R. Datko. Uniform asymptotic stability of evolutionary processes in a Banach space. SIAM J. Math Anal. 3 (1973), 428- 445.

[5] R. Datko. Not all feedback stabilized hyperbolic systems are robust with respect to small time delays in their feedbacks. SIAM J. Control Optim. 26 (1988), 697-713.

[6] W. Day. The decay of the energy in a viscoelastic body. Mathematics 27 (1980), 268-286.

[7] W. Desh and K. B. Hannsgen, Y. Renardy and R. L. Wheeler. Boundary stabilization of an Euler-Bernoulli beam with viscoelastic damping. Proceedings of the 26th IEEE CDC Conference, Los Angeles, (1987).

[8] W. Desh and R. K. Miller.Exponential stabilization of Volterra integrodifferential equations in Hilbert spaces. J. Differential equations 70 (1987), 366-389.

[9] G. Duvaut and J. Lions. Les Inéquations en mécanique et en Physique. Paris: Dunod. (1969).

[10] K. B. Hannsgen and R. L. Wheeler. Time delays and boundary feedback stabilization in one-dimensional viscoelasticity. In distributed parameter systems eds. F. Kappel and K. Kunish. pp 136-152. 3rd International Conference Vorau 1986 (Berlin: Springer, 1987).

[11] J. E. Lagnese and J. L. Lions. Modeling, Analysis and control of thin plates. (Paris: Masson, 1987).

[12] R. C. MacCamy and J.S.W. Wong. Exponential stability for a nonlinear functional differential equations. J. Math. Anal. Appl. 39 (1972), 609-705.

[13] J. E. Muñoz Rivera, Maria Grazia Naso; Asymptotic stability of semigroups associated to linear weak dissipative systems with memory. Mathematical Analysis and Applications Vol 326 (1), (2007), 697 - 707.

[14] J. Pruss. Positivity and regularity of hyperbolic Volterra equation in Banach spaces. Math. Ann. 279 (1987), 317-344.

[15] Y. S. Santiago Ayala. About decay of solution of the wave equation with dissipation. PROYECCIONES. Vol 26 Num 1 (2007) 37-71.

[16] Y. S. Santiago Ayala, J. E. Muñoz Rivera; Exponential decay for a Von Karman System with memory. Advances in differential equations Vol 9 Num 9-10 (2004), 1115-1142.

[17] Y. S. Santiago Ayala. Sobre la analiticidad del semigrupo Co asociado a un sistema viscoelástico. PESQUIMAT Vol VI Num 2 (2003), 27-36.

[18] R. Showalter. Hilbert Space Methods for Partial Differential Equations . London: Pitman, (1977) 